\section{Embolization of Brain Arteriovenous Malformations for Cure: Because We Could and Because We Should}

We read with great interest the commentary by Jayaraman and Cloft ${ }^{1}$ about the necessity for curative embolization of brain arteriovenous malformations (AVMs), and we feel obliged to present our objections to their views.

It is absolutely true that for any treatment to be justified, it needs to have high success and low complication rates. Because embolization for brain AVMs can be performed either as an adjunctive (preoperative or preradiosurgical) measure or as a definite treatment, it is natural that these rates depend on the initial planning and on the strategy of the treating physicians. When treating physicians are pursuing total cure, higher total occlusion and higher complication rates may result. On the other hand, when the physicians feel that they can discontinue the embolization at the first difficulty and refer the patient for surgery or radiosurgery to treat the remnant, the complication rate is, of course, lower as well as the total occlusion rate. Moreover, when additional treatments are applied after a partial embolization, the patients are exposed to the additional risks of those treatments as well. Therefore, any comparison between series of curative embolization with Onyx (ev3, Irvine, Calif) and series of adjunctive embolization with Onyx or $n$-butyl 2-cyanoacrylate ( $n$-BCA) is not justified.

In analyzing the recent studies on brain AVM embolization, Jayaraman and $\mathrm{Cloft}^{1}$ made an inexplicable mistake in presenting the total occlusion rates. In our recent study ${ }^{2}$ and that of Mounayer et al, ${ }^{3}$ the rates of total occlusion were $53.9 \%$ and $49 \%$, respectively. In this commentary, they are reported as $27.7 \%$ and $28 \%$ because its authors calculated these rates as the percentage of patients with total cure of the total population of patients in the studies, omitting to subtract from the total population the number of patients who are still receiving treatment and are scheduled for more embolization sessions. It is clear that in these patients (49/101 in our series), the treatment has not been concluded, further occlusion of their AVMs could be achieved, and some could even reach total occlusion. Therefore, considering that the method has failed to provide total cure in these patients and including them in the calculation of the total occlusion rate and the evaluation of the method's success is obviously wrong and unnecessarily discrediting. The footnote of the table in the commentary that "further endovascular treatment was planned in some patients" is misleading and certainly does not allow the reader to discover the actual total occlusion rates.

Jayaraman and $\mathrm{Cloft}^{1}$ surprisingly dismiss partial embolization of an AVM as a factor leading to better surgical and radiosurgical results in the treatment of the remnant because of a "lack of evidence." We believe that the excellent study by van Rooij et al ${ }^{4}$ offers more than sufficient evidence and is in accordance with our own and with the international experience in the microsurgical resection of partially embolized AVMs.

The authors of the comment also focus on the matter of small AVMs, implying that it is unethical for patients to be treated with an endovascular method that has a success rate "no better than $30 \%$, or even as high as $50 \%$ " with a complication rate of " $7 \%$ to $18 \%$." In comparison, they state that surgery for small AVMs can provide $100 \%$ cure with complications ranging from $0 \%$ to $15 \%$, whereas radiosurgery offers $70 \%$ cure with complications less that $10 \%$. This comparison is not justified because the authors compare apples with oranges: the cure and complication rates of embolization for the total population of patients with AVM (harboring small, medium, and large
AVMs of all Spetzler-Martin grades) with the results of surgery and radiosurgery only in small AVMs. In our study, ${ }^{2}$ we did not report on the results of AVM treatment stratified by size, location, or SpetzlerMartin grades. Had we done so, it would seem that in small AVMs $(<3 \mathrm{~cm})$ in "noneloquent" areas of the brain, we achieved a total occlusion rate of more than $95 \%$ with a complication rate less than $2 \%$ (including groin hematomas). We are certain that this is also the experience of other teams performing curative embolization of AVMs. These results are far superior than those of surgery or of radiosurgery, but other factors must also be taken into account when deciding on the best treatment for a specific patient: patient discomfort and duration of disability; the influence of comorbidities; and, finally, the patient's personal preference.

Patients treated with embolization for a small AVM can be discharged from the hospital the next day and can resume their normal life activities in a few days, whereas those treated with craniotomy must be hospitalized for at least 4 to 5 days and may take up to 1 month to convalesce completely. Craniotomy in elderly patients with severe comorbidities can result in lengthy hospitalization or even serious complications. These setbacks may be avoided with endovascular treatment.

One important factor that we seem to neglect in the age of "evidence-based medicine" is the personal views of the patient regarding the choice of treatment. In all of our patients, we had presented the 4 treatment options (observation, embolization, surgery, and radiosurgery). Confronted with an AVM that may have a low bleeding rate but that is potentially lethal, not even 1 patient opted for no treatment or for either surgery or radiosurgery alone. Patients seem to have the common sense to choose a minimally invasive therapy that can provide cure with a reasonable complication rate, but it can be complemented by other methods should it fail to provide total obliteration of the AVM.

Finally, Onyx may seem to the authors of this comment as a "shiny new toy," but in our hands and in those of others, it has yielded far superior results than $n$-BCA embolization. In our view, it is another tool in our armamentarium that combined with other materials ( $n$ BCA, particles, or even coils) in certain cases, as we reported in our study, ${ }^{2}$ can offer the possibility for significantly better results in the embolization of brain AVMs.

\section{References}

1. Jayaraman M, Cloft HJ. Embolization of brain arteriovenous malformations for cure: because we could or because we should? AJNR Am J Neuroradiol 2009;30:107-08

2. Katsaridis V, Papagiannaki C, Aimar E. Curative embolization of cerebral arteriovenous malformations (AVMs) with Onyx in 101 patients. Neuroradiology 2008;50:589-97

3. Mounayer C, Hammami N, Piotin M, et al. Nidal embolization of brain arteriovenous malformations using Onyx in 94 patients. AJNR Am J Neuroradiol 2007; $28: 18-23$

4. van Rooij WJ, Sluzewski M, Beute GN. Brain AVM embolization with Onyx. AJNR Am J Neuroradiol 2007;28:172-77

V. Katsaridis

C. Papagiannaki

E. Aimar

Unit for Endovascular Neurosurgery Neurosurgical Department Papanikolaou General Hospital Thessaloniki, Greece

DOI 10.3174/ajnr.A1458

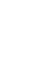

